\title{
Exploradores en acción: la yincana como elemento motivador y socializador en la enseñanza de ELE
}

CRistina Villalba

Universitat de València - Universitat Jaume I

cristina.villalba@uv.es

\begin{abstract}
Resumen: El objetivo de este artículo es compartir una experiencia didáctica diseñada para trabajar el componente sociocultural y afectivo en contextos de inmersión para el aprendizaje de español como lengua extranjera. La razón: la necesidad de facilitar el proceso de adaptación del estudiante recién llegado a un contexto de inmersión en lengua y cultura española. Esta propuesta ofrece, en primer lugar, informaciones básicas de la ciudad (Valencia - barrio del Carmen), para que pueda orientarse por su zona de residencia durante el curso y se trabaje el componente sociocultural. En segundo lugar, favorece la comunicación con hablantes nativos, de modo que pueda poner en práctica sus conocimientos lingüísticos hasta el momento y naturalice la comunicación con hablantes nativos más allá de su nivel de competencia. Por último, se estimula el proceso de socialización a partir del desarrollo de tareas por grupos en las que los participantes puedan superar las limitaciones individuales gracias al apoyo del grupo.
\end{abstract}

Palabras clave: gamificación, ludificación, yincana, Valencia, E/LE

Explorers in action: the gymkhana as a motivating and socializing device for teaching SFL

Abstract: The main purpose of this paper is to share a didactic experience designed to work on the sociocultural and affective component in immersion contexts for Spanish as a foreign language. The reason that motivated this proposal, a gymkhana, arises from the need to facilitate the adaptation process of the newly arrived student. For this reason, this proposal offers, in the first place, basic information about the city (Valencia - el Carmen neighbourhood). This way, the student will be able to navigate in his/her area of residence during the course and work on the socio-cultural component. Secondly, it favours the communication with native speakers, so that they can put into practice their linguistic knowledge and naturalize the fact of communicating with native speakers beyond their level of competence. Finally, the socialization process is stimulated by the development of tasks by groups in which the participants can overcome individual limitations thanks to the support of the rest of the members.

Keywords: gamification, gymkhana, Valencia, E/LE

\section{Introducción}

El proceso de aprendizaje de E/LE en un contexto de inmersión supone un triple reto para el estudiante. En primer lugar, debe enfrentarse a un nuevo espacio, que generalmente no le es familiar, y tratar de desenvolverse con naturalidad. En segundo lugar, este nuevo espacio genera una serie de situaciones que le obligan a comunicarse en una lengua en la que todavía no se siente plenamente competente. Y, en tercer lugar, el traslado a este 
espacio supone la pérdida del contacto directo con familiares, amigos y conocidos, que se encuentran en su país de origen. En consecuencia, no es extraño que, durante los primeros días en el nuevo país, el estudiante se sienta desamparado y desorientado y que esto pueda afectar, a su vez, a su predisposición a comunicarse.

Una manera de mitigar estos sentimientos y fomentar la predisposición a comunicarse es generar actividades lúdicas desde el aula y fuera de ella para favorecer que el estudiante se relacione con el nuevo espacio al tiempo que crea vínculos con sus compañeros. En esta línea, en este artículo se presenta una experiencia práctica diseñada para estudiantes estadounidenses de niveles A1-B2 dentro de un programa de inmersión que tiene como contexto la ciudad de Valencia.

\section{Marco teórico}

El componente lúdico en la enseñanza destaca como uno de los recursos más motivadores para los estudiantes. Así, diversos autores destacan el papel de la ludificación (o gamificación, si se toma el préstamo del inglés) como una poderosa herramienta a disposición del docente para enriquecer la experiencia de aprendizaje mediante el juego (Mora 2013). Son muchas las ventajas que se obtienen a partir de la utilización de elementos propios del juego como las tarjetas o la competición. Foncubierta y Rodríguez (2014) explican que la introducción de juegos relacionados con los contenidos puede contribuir a captar la atención, mejorar sus capacidades memorísticas y favorecer la adquisición de habilidades y conocimientos de nuestros alumnos.

Estos autores también destacan que en el plano afectivo, el empleo de juegos en la enseñanza de segundas lenguas también desarrolla un papel destacado, pues ayuda al desarrollo del autoconcepto y a la socialización del estudiante. La dependencia positiva que se crea entre los miembros de los equipos genera un sentimiento de pertenencia, de modo que el estudiante se siente respaldado por el grupo. Al mismo tiempo, el estudiante también se siente capaz de superar las limitaciones individuales al contar con otros participantes que tienen habilidades y conocimientos diferentes a los suyos. Se crea, de este modo, un espacio perfecto para el aprendizaje cooperativo y, al mismo tiempo, se reduce el sentimiento de soledad o desamparo que puede sentir el estudiante que se traslada a otro país para aprender un idioma.

Otra manera de facilitar la acogida del estudiante y que también supone un elemento motivador es emplear recursos que conecten con la realidad del estudiante. En el caso del alumnado joven adulto, es común que estos compartan sus experiencias a través de fotos o vídeos en redes sociales, de manera que estas plataformas se convierten igualmente en medios de socialización y comunicación aprovechables para mejorar destrezas (Villalustre y Del Moral 2015; Cordeiro, 2018). Por este motivo, la propuesta que presentamos a continuación se apoya en el componente visual y en compartir experiencias en las redes sociales como una manera de involucrar al estudiante en la actividad.

Por lo que respecta al plano lingüístico, hay que tener en cuenta que el estudio de una segunda lengua en un contexto de inmersión supone que el estudiante va a estar continuamente expuesto a un input en una lengua cuyo nivel de competencia es inferior a la de su lengua de origen. Este hecho puede generar frustración (especialmente durante los primeros días de estancia) llegando a mermar las capacidades para comunicarse. Es por ello que la elaboración de actividades lúdicas, especialmente en situaciones reales, puede contribuir a reducir el nivel de estrés y facilitar que el estudiante se comunique en 
español. Por esta razón, en esta propuesta didáctica combinamos actividades que explotan las destrezas integradas, incluyendo momentos de interacción oral con hablantes nativos, la comprensión visual-espacial y la comprensión lectora.

Además, el contexto y la situación son considerados la primera causa de variabilidad en cuanto al ritmo de aprendizaje y el grado de perfección que el aprendiz pueda alcanzar (Martín Martín 2004: 270). De esta manera, los contextos de inmersión cuentan con la ventaja de que los espacios donde el estudiante tiene que interactuar en la segunda lengua son más numerosos y van más allá del ámbito académico. Existe, por tanto, una necesidad natural de comunicarse que es, a la vez, una presión añadida, pues no es lo mismo comunicarse con otros estudiantes que tienen el mismo nivel de español que exponerse a hablantes nativos.

\section{Experiencia didáctica: Yinacana en Valencia}

La experiencia didáctica que se presenta a continuación es una yincana. La yincana es una modalidad de juego en el que los participantes deben realizar una serie de pruebas en varios espacios antes que sus compañeros. En nuestro caso, los estudiantes contaban con una serie de preguntas que debían responder buscando en distintas localizaciones marcadas en unos mapas. Para garantizar que los estudiantes habían estado allí, debían hacerse, además, una foto.

Esta yincana fue diseñada para un grupo de estudiantes procedentes de una universidad estadounidense que vinieron a Valencia a realizar un curso intensivo de español. Los niveles de estos estudiantes oscilaban entre el A1 y el B2 y solo algunos se conocían entre sí, pues cada uno estudiaba distintos grados. Buena parte de estos estudiantes se alojaban en la zona del casco histórico de Valencia, de modo que esta actividad se desarrolló para que conocieran mejor aquel barrio, para trabajar el componente sociocultural y para dar a conocer algunas curiosidades del barrio del Carmen, de la ciudad de Valencia y del país. Otro de los motivos que nos llevaron a seleccionar este espacio es que, además de ser una de las zonas turísticas por excelencia de la ciudad, apenas hay tráfico y los estudiantes podían moverse con más libertad.

Asimismo, a partir de las actividades de la yincana también se quiso trabajar la predisposición a comunicarse, pues se trata de que los estudiantes tomen un primer contacto con los hablantes nativos y que se familiaricen con los intercambios comunicativos en español. En la misma línea, también se gestiona el componente social y afectivo trabajando en grupos de distintos niveles. De este modo, cada estudiante puede conocer a otros compañeros, compartir con ellos sus habilidades y conocimientos y superar sus propias limitaciones gracias al conocimiento y habilidades de los demás.

\subsection{Agrupaciones}

Como se trata de la actividad inicial del curso, lo más natural es que, instintivamente, los estudiantes busquen a aquellas personas que conocen de antes de comenzar el curso o que se queden con las personas que pertenecen al nivel en el que han sido organizados. Como este último hecho puede suponer un desequilibrio para la realización de la actividad, pues las personas con niveles más bajos van a tener más dificultades para comprender y realizar las tareas, lo ideal es que se favorezca una agrupación en donde se incluyan participantes de distintos niveles. Para ello se pueden seguir distintas dinámicas como la agrupación a 
través del uso de canciones o repartiendo pegatinas de diferentes colores, por citar algunos ejemplos.

En cuanto al número de miembros por agrupación, lo ideal es que el grupo esté compuesto por cuatro, cinco o seis personas como máximo. Dentro del grupo, se debe decidir quién será el capitán o capitana del equipo, el nombre que utilizarán para identificarse y un lema.

\subsection{Materiales}

Como ya se ha explicado, la yincana consiste en la resolución de unas preguntas a partir de la búsqueda de la respuesta en distintos enclaves y la toma de una fotografía del grupo. Los materiales que necesitan para realizar la yincana son los mapas (que pueden consultarse a continuación), un teléfono móvil para hacer fotografías, acceso a internet y una red social para que el docente pueda hacer un seguimiento de los progresos de los estudiantes a medida que suben las fotos. En nuestro caso, el capitán o capitana del equipo tenía la responsabilidad de subir las fotografías a su cuenta de Instagram con la etiqueta \#descubriendoValencia. No obstante, también se puede realizar esta actividad utilizando otras aplicaciones como Whatsapp, Telegram, Facebook o correo electrónico, por citar algunos ejemplos donde los grados de privacidad para ver las fotografías varían.

Finalmente, para evitar que los grupos aprovechen la información del resto de estudiantes, se recomienda alterar el orden de las pruebas, de modo que no coincidan varios grupos en el mismo espacio.



Imagen 1. Prueba 1 


\section{PRUEBA 2 Puerta de Serranos}

La Puerta de Serranos es una de las puertas de la antigua muralla de Valencia. Se construyeron a finales del siglo XIV y hoy es uno de los edificios más característicos de la ciudad. ¿Cuántas torres hay en este edificio?

\#descubriendoValencia $\rightarrow$ haceos una foto en la que se vea la fachada del edificio



Imagen 2. Prueba 2

\section{PRUEBA 3 La plaza del Negrito}

Esta fue la primera fuente de agua natural de la ciudad. Se inauguró en 1850 y la gente iba a esa fuente para obtener agua potable. ¿Qué sostiene "el negrito"?

\#descubriendoValencia $\rightarrow$ haceos una foto en la que se vea la fuente

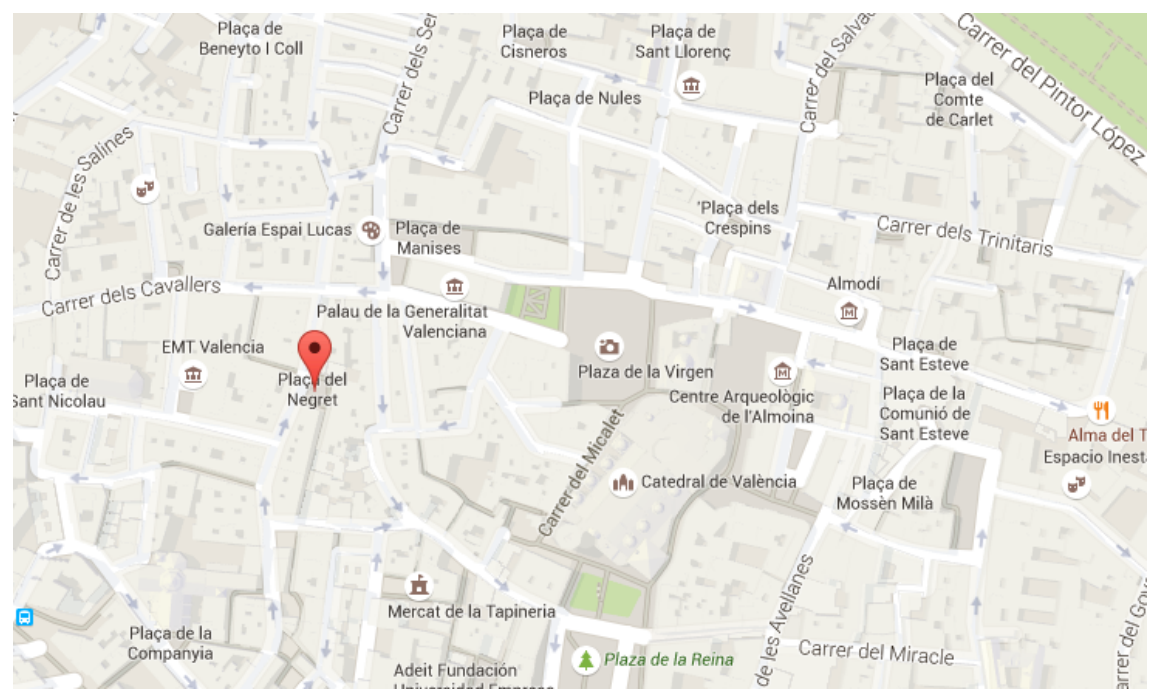

Imagen 3. Prueba 3 


\section{PRUEBA 4 La fachada más pequeña de Europa}

Valencia tiene el honor de tener la casa con la fachada más estrecha de Europa. Este edificio se encuentra en la plaza Lope de Vega. ¿Sabéis en qué número?

\#descubriendoValencia $\rightarrow$ haceos una foto en la puerta del edificio

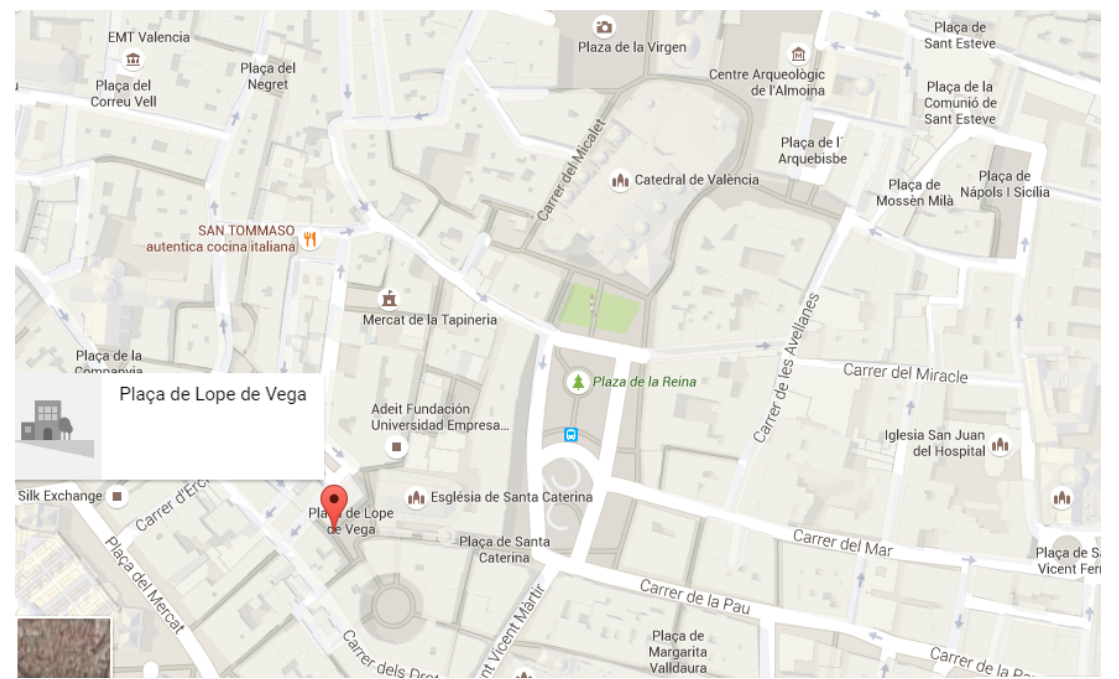

Imagen 4. Prueba 4

\section{PRUEBA 5 Vacas y horas}

Seguro que te has topado con alguna de estas vaquitas al pasear por Valencia (u otras ciudades españolas). La primera tienda ALE-HOP se fundó en Ondara (Alicante) en 2001 y hoy cuenta con muchas tiendas. Entra a la tienda y pregunta por el horario comercial. \#descubriendoValencia $\rightarrow$ haceos una foto con la vaca de la entrada

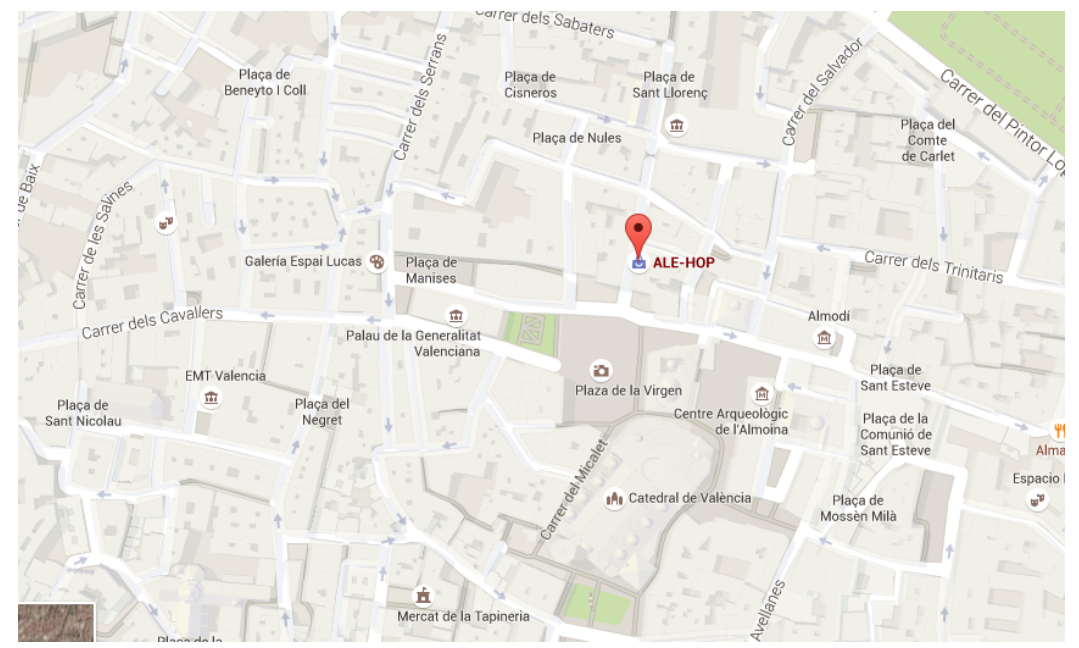

Imagen 5. Prueba 5 


\section{PRUEBA 6 Las acequias del río Turia}

¿Habéis visto la fuente de la Plaza de la Virgen? El hombre que aparece en el centro representa al río Turia y las mujeres (ninfas) que hay a su alrededor se identifican con las acequias que riegan la huerta de Valencia. Anota los nombres de las acequias de la ciudad.

\#descubriendoValencia $\rightarrow$ haceos una foto con la fuente



Imagen 6. Prueba 6

\section{PRUEBA 7 Los escalones de EI Miguelete}

Dicen que una de las vistas más bonitas de la ciudad se puede ver desde lo alto del campanario "El Miguelete". Entrad a la catedral por la puerta que aparece marcada en el mapa y preguntad cuántos escalones tiene este campanario y por qué se llama el campanario "Miguelete" o "Micalet".

\#descubriendoValencia $\rightarrow$ haceos una foto en la entrada de la catedral

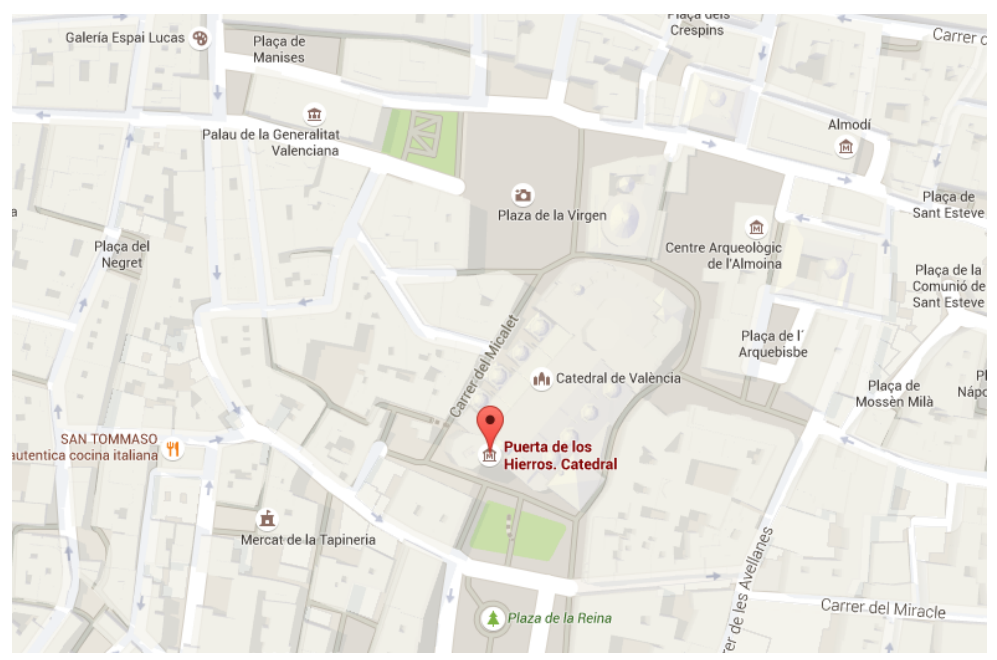




\section{PRUEBA 8 La Lonja de la Seda}

Uno de los edificios más hermosos de la ciudad es el de la Lonja. Este edificio tiene un jardín con unos árboles característicos de Valencia. Id al punto que aparece en el mapa y preguntad al guardia de la entrada qué árboles hay en el jardín.

\#descubriendoValencia $\rightarrow$ haceos una foto en la entrada de la lonja

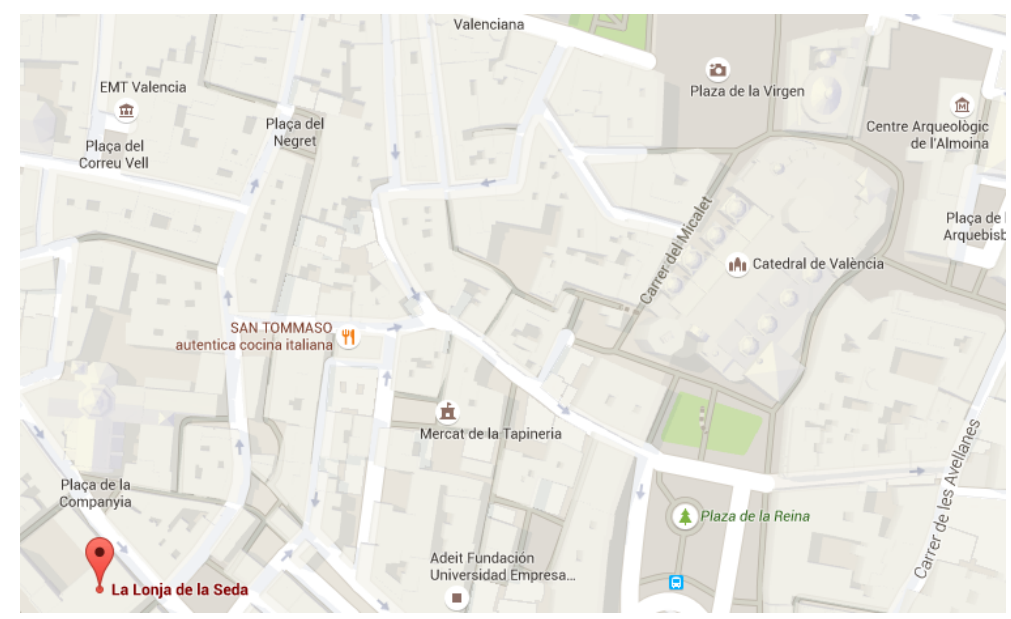

Imagen 8. Prueba 8

\section{PRUEBA 9 Los helados más famosos de Valencia}

¿Estáis cansados y os apetece tomar algo fresco? Esta es vuestra prueba. Esta heladería es una de las más antiguas de Valencia y se la conoce por los sabores de helados "curiosos" que tienen. Anotad el sabor más raro que encontréis.

\#descubriendoValencia $\rightarrow$ haced una foto del helado con el sabor más raro que encontréis

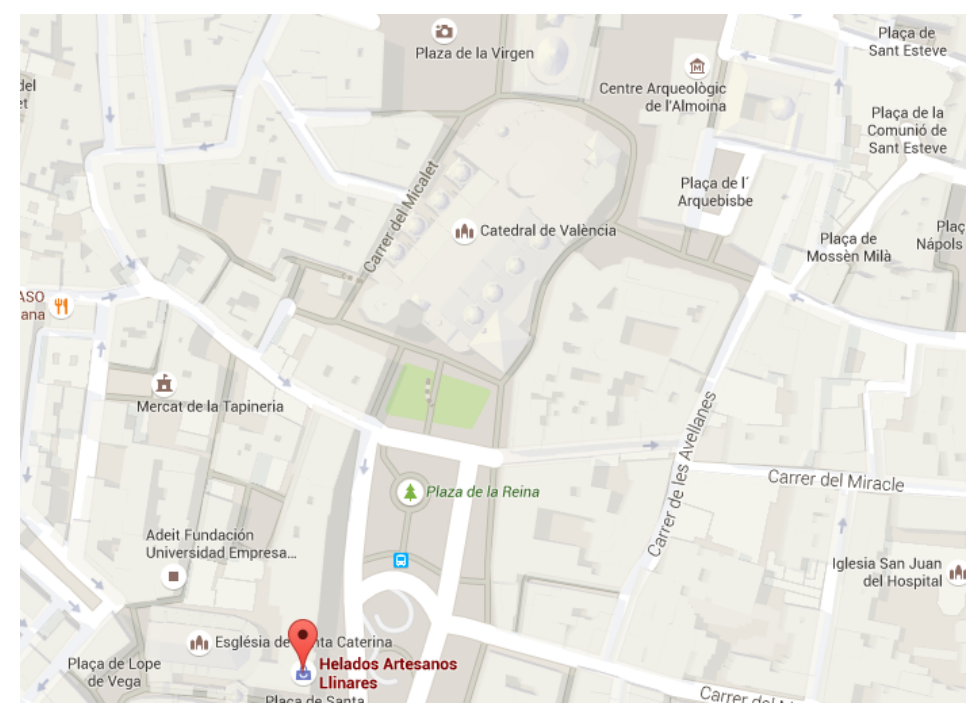

Imagen 9. Prueba 9 
La yincana finaliza cuando los estudiantes ya han realizado todas las pruebas y se reúnen en el punto de salida con el profesor. Este momento se puede aprovechar para premiar a los ganadores (por ejemplo, con una insignia de explorador/a de Valencia) comparar respuestas, resolver dudas, y reflexionar sobre la experiencia. También es el momento de identificar las posibles dificultades que los alumnos han tenido en su comunicación, de modo que esta reflexión puede convertirse en una herramienta útil para conocer las carencias de los alumnos y proyectar actividades destinadas a solventarlas durante el curso.

\subsection{Variaciones de la actividad}

Esta actividad puede modificarse de distintas maneras para adaptarlo a otras zonas y ciudades. Si se quiere introducir contenidos de lengua además del componente sociocultural, es posible proponer ejercicios que deben realizar los estudiantes en los distintos emplazamientos. En este sentido, las nuevas tecnologías permiten crear códigos QR que el docente puede pegar en los lugares designados de modo que solo los estudiantes los reconozcan y puedan acceder a los contenidos.

Otra opción es que, a medida que se acerca el final de curso, sean los propios estudiantes los que ayuden a elaborar la yincana con sus rincones favoritos de la ciudad. Así, cada grupo tendría que investigar por su cuenta, lo que favorece el trabajo cooperativo, para proponer dos lugares y preparar algún ejercicio o prueba relacionado con la ubicación. El profesor revisaría la propuesta con los estudiantes y, posteriormente, podría realizar la yincana con los retos de los alumnos.

\section{Conclusión}

A modo de conclusión esta experiencia didáctica evidencia la validez de aprovechar las oportunidades que el contexto de inmersión ofrece a los estudiantes más allá del espacio del aula. Por esta razón, la realización de una yincana al principio del curso es muy positiva, puesto que es una actividad semiguiada que permite al alumno familiarizarse con el entorno al tiempo que le prepara para explorar la ciudad y sentirse más cómo en el nuevo espacio. Igualmente, se incentiva a los alumnos a aprovechar todas las oportunidades para desenvolverse utilizando la lengua meta en situaciones cotidianas. En este sentido, el apoyo del grupo es imprescindible para aumentar la confianza de los estudiantes en su competencia comunicativa y desarrollar en el aula las estrategias para superar las dificultades lingüísticas y socioculturales que pueden surgir. La experiencia de la yincana fue muy fructífera y refuerza la importancia de ofrecer experiencias de aprendizaje memorables a los estudiantes en las que ellos participan activamente. Asimismo, la inclusión de elementos lúdicos favorece la participación de los alumnos a la vez que disminuye el desfase entre los niveles, de manera que se aumenta la disposición de los alumnos a expresarse en la lengua meta.

\section{Bibliografía}

CORDEIRO, D. M. (2018). Las inteligencias múltiples como herramienta para mejorar las destrezas orales en el aula de Español como Lengua Extranjera: una investigación acción. València: Universitat de València. 
FonCUBIERTA, J. M., Y RodRíGUEZ, C. (2014). «Didáctica de la gamificación en la clase de español». PDP ELE, 1-8. Disponible en línea.

MARTín MARTíN, J. M. (2004). «La adquisición de la lengua materna (L1) y el prendizaje de una segunda lengua (L2) / lengua extranjera (LE): procesos cognitivos y factores condicionantes». En Vademécum para la formación de profesores (pp. 261-286). Madrid: SGEL.

MorA, F. (2013). Neuroeducación: sólo se puede aprender aquello que se ama. Madrid: Alianza.

Villalustre, L., Y Del Moral, M. (2015). «Gamificación: Estrategia para optimizar el proceso de aprendizaje y la adquisición de competencias en contextos universitarios». Digital Education Review, 27, 13-31. 